\title{
Efficacy and safety of immune checkpoint inhibitors in gastric cancer: a network meta-analysis of well-designed randomized controlled trials
}

\author{
Siwei Pan ${ }^{1,2}$, Kai $\mathrm{Li}^{1,2}$, Baojun Huang ${ }^{1,2}$, Jinyu Huang ${ }^{1,2}$, Huimian $\mathrm{Xu}^{1,2}$, Zhi Zhu ${ }^{1,2}$ \\ ${ }^{1}$ Department of Surgical Oncology, First Hospital of China Medical University, Shenyang, China; ${ }^{2}$ Department of Surgical Oncology and General \\ Surgery, Key Laboratory of Precision Diagnosis and Treatment of Gastrointestinal Tumors, Ministry of Education, The First Affiliated Hospital of \\ China Medical University, Shenyang, China \\ Contributions: (I) Conception and design: Z Zhu, H Xu; (II) Administrative support: Z Zhu, H Xu; (III) Provision of study materials or patients: Z \\ Zhu, S Pan; (IV) Collection and assembly of data: S Pan, K Li; (V) Data analysis and interpretation: S Pan, B Huang, J Huang; (VI) Manuscript \\ writing: All authors; (VII) Final approval of manuscript: All authors. \\ Correspondence to: Zhi Zhu, MD, PhD; Huimian Xu, MD, PhD. Department of Surgical Oncology, the First Affiliated Hospital of China Medical \\ University, North Nanjing Street 155, Shenyang 110001, China. Email: zhuzhi@cmu.edu.cn; xuhuimian@126.com.
}

\begin{abstract}
Background: Immune checkpoint inhibitors (ICIs) that inhibit the programmed death 1 (PD-1)/ programmed death-ligand 1 (PD-L1) and cytotoxic T-lymphocyte antigen 4 (CTLA-4) interactions have shown promising prospects as treatment options for advanced gastric cancer (AGC). This manuscript analyzed well designed clinical trials to evaluate the efficacy and safety of immunotherapy in AGC.

Methods: PubMed, Embase, the Cochrane Library, and Medline were searched for randomized controlled trials (RCTs) of AGC treatments that were published before April 2020. Progression-free survival (PFS), overall survival (OS), objective response rate (ORR), and treatment-related adverse events (TRAEs) were evaluated to determine the efficacy and safety of ICIs. Network meta-analysis was performed using a random-effects model under the Bayesian framework. The ability of each treatment was ranked using the surface under the cumulative ranking (SUCRA) curve.

Results: Our analysis included five studies having seven immunotherapy regimens and 1,730 patients. The network meta-analysis showed that nivolumab $1 \mathrm{mg} / \mathrm{kg}$ every 3 weeks plus ipilimumab $3 \mathrm{mg} / \mathrm{kg}$ every 3 weeks $(88.369 \%)$ was the regimen most likely to improve PFS. Nivolumab $3 \mathrm{mg} / \mathrm{kg}$ every 3 weeks $(84.563 \%)$ and nivolumab $1 \mathrm{mg} / \mathrm{kg}$ every 3 weeks plus ipilimumab $3 \mathrm{mg} / \mathrm{kg}$ every 3 weeks $(84.556 \%)$ were similarly best for OS outcome with excellent tolerance. The regimen of avelumab $10 \mathrm{mg} / \mathrm{kg}$ every 2 weeks $(91.167 \%)$ had the lowest TRAEs. All immunotherapies had similar response rates.
\end{abstract}

Conclusions: We recommend nivolumab $3 \mathrm{mg} / \mathrm{kg}$ every 2 weeks or nivolumab $1 \mathrm{mg} / \mathrm{kg}$ every 3 weeks plus ipilimumab $3 \mathrm{mg} / \mathrm{kg}$ every 3 weeks as the preferred regimen due to their high efficacies.

Keywords: Advanced gastric cancer (AGC); chemotherapy; immunotherapy; network meta-analysis

Submitted Sep 26, 2020. Accepted for publication Nov 27, 2020.

doi: 10.21037/atm-20-6639

View this article at: http://dx.doi.org/10.21037/atm-20-6639

\section{Introduction}

Gastric cancer (GC) has a particularly poor prognosis and a high incidence rate worldwide (1). Of the total cases of GC reported worldwide in 2018, China's cases accounted for $>45 \%$ of the incidence rate and $>50 \%$ of the mortality rate. D2 radical surgery is still the most effective treatment for advanced gastric cancer (AGC) (2). Despite remarkable improvements in surgical and comprehensive therapies, recurrence and metastasis are still the main causes of death from AGC.

Treatments aim to stabilize disease progression, improve 
patients' prognosis, and reduce recurrence and metastasis rates for AGC. In recent years, the therapeutic strategy for GC has shifted from surgical treatment to comprehensive treatment based on collaboration between members of a multi-disciplinary team (3). With the development of adjuvant/neoadjuvant therapy, as well as progress in targeted therapy and immunotherapy drugs, the 5-year survival rate of GC patients in China has improved (4). First-line chemotherapy, which is usually comprised of platinum and fluoropyrimidine, can extend the overall survival (OS) by approximately 7 months.

For patients with progression of disease on first-line chemotherapy, treatment options include chemotherapy with irinotecan, taxanes (paclitaxel or docetaxel), and trastuzumab for HER-2 (epidermal growth factor receptor 2), or ramucirumab [a monoclonal antibody against vascular endothelial growth factor receptor 2 (VEGFR2)] used either in monotherapy or in combination with paclitaxel (5). However, most AGC patients experience disease progression following these treatments. No guidelines have been recommended for the standard treatment for patients who have failed two or more lines of therapy. Nonetheless, the prognosis for these patients remains poor. Novel therapy options with acceptable safety profiles need to be developed.

Immune checkpoint inhibitors (ICIs) offer promising new treatment options for AGC patients. Inhibiting immune checkpoints can increase $\mathrm{T}$ cell activity and enhance antitumor immunity (6). There are currently six FDA-approved ICI immunotherapy options for GC. These include humanized monoclonal antibodies against programmed death 1 (PD-1) (nivolumab, pembrolizumab), programmed death-ligand 1 (PD-L1) (avelumab, atezolizumab, durvalumab), and cytotoxic T-lymphocyte antigen 4 (CTLA-4) (ipilimumab) (7). Several trials investigating the effects of immunomodulating agents and chemotherapies on AGC have been performed and have shown good safety and a positive effect on the survival of patients (8-10). However, due to the limited number of trials, no standard guideline is currently available. Many challenging issues remain, including understanding the specific immunogenicity of gastric carcinoma, selecting the best regimen and the optimal protocol for combining chemotherapy with immunotherapy, optimizing longterm survival with multi-agent cancer immunotherapy combination regimens, and personalizing approaches through composite biomarkers. Furthermore, additional phase III studies on ICIs for metastatic GC have shown discordant results. So far, we failed to find a robust study for patients with AGC that compares the effects of treatment selection in clinical practice.

Traditional meta-analysis uses data only of clinical trials to directly analyze the comparison in the study, but cannot do an indirect analysis between multiple treatment methods among trials with different treatments. Hence, in the present study, we performed a network meta-analysis of well-designed clinical trials to evaluate the impact of ICIs on the outcome of patients with AGC, considering the predictors of efficacy and safety. We present the study in accordance with the Preferred Reporting Items for Systematic Reviews statement for Network Meta-Analyses (PRISMA-NMA) reporting checklist (11) (available at http://dx.doi.org/10.21037/atm-20-6639).

\section{Methods}

\section{Literature search}

We searched the PubMed, Embase, the Cochrane Library, and Medline databases for intake-related studies that were published between January 1, 2005, and April 11, 2020. The search keywords in the search strategy (provided in detail in Appendix 1) referred to GC, immunotherapy, immune checkpoints (PD-1, PD-L1, and CTLA-4), and specific drug names (ipilimumab, nivolumab, pembrolizumab, sintilimab, camrelizumab, atezolizumab, durvalumab, avelumab, and toripalimab). Two authors (SP and ZZ) independently assessed the process of the literature search and the full texts for eligible inclusions.

\section{Study selection}

Publications adopted in this network meta-analysis met the criteria as follows: (I) prospective randomized controlled trials (RCTs); (II) patients were proven to have GC or including gastroesophageal junction (GEJ) cancer; (III) pairwise comparison of treatment modalities, including ICIs; and (IV) inclusion of one or more of the following outcomes: progression-free survival (PFS), OS, objective response rate (ORR), or treatment-related adverse events (TRAEs). Trials that included ICIs in the first-line therapy were excluded. If trials had multiple publications, the latest publication was adopted to provide the longest follow-up.

\section{Data extraction and quality assessment}

The following information was extracted from the eligible 
studies by two independent authors: first author's name, abbreviation of study, publication year, clinical register, country, sample size, age, intervention regimen, control treatment, and follow-up. PFS was set as the primary outcome of the current analysis; the secondary outcomes were OS and severe TRAEs (grades 3 to 5 according to the National Cancer Institute Common Terminology Criteria for Adverse Events). All eligible studies were evaluated for risk of bias using the Cochrane Risk of Bias tool, which assigns three levels of bias across each of seven aspects (12). Disagreements in the above parts were resolved through group discussions.

\section{Statistical analysis}

PFS and OS were measured using hazard ratios (HRs) and $95 \%$ confidence intervals (CIs), which could reduce the heterogeneity caused by the different follow-ups that were extracted from each adopted study. Odds ratios (ORs) with $95 \%$ CIs were also calculated in the analyses for ORR and TRAE outcome. Assessments of efficacy and safety of immunotherapy compared with placebo/chemotherapy were carried out initially by traditional pairwise meta-analysis in RevMan software version 5.3. Statistical heterogeneity was assessed in each comparison using the $\mathrm{I}^{2}$ statistic and $\mathrm{P}$ value. The random-effect model was adopted when $\mathrm{I}^{2}>50 \%$, otherwise the fixed-effect model was adopted. Next, the network meta-analyses of two classifications were carried out in a Bayesian framework in OpenBUGS version 3.2.3. One was based on the different drugs and doses; the other was based on the different immune targets. We did not carry out an inconsistency analysis since paired comparisons in the only closed loop was from the same study. Surface under the cumulative ranking (SUCRA) scores were used to rank treatments for each outcome and calculated by $\mathrm{R}$ software version 3.5.3. A higher SUCRA score stands for better efficacy (13). All tests were two-sided with an $\alpha$ of 0.05 .

\section{Results}

\section{Included studies and their characteristics}

Five RCTs, which included 1,730 patients, were ultimately incorporated into this network meta-analysis after the systematic literature retrievals $(10,14-17)$. The filtering process is illustrated in the flow diagram (Figure 1). Among the five RCTs, patients received seven different treatments: placebo/chemotherapy, nivolumab $3 \mathrm{mg} / \mathrm{kg}$ every 2 weeks, avelumab $10 \mathrm{mg} / \mathrm{kg}$ every 2 weeks, pembrolizumab $200 \mathrm{mg}$ every 3 weeks, ipilimumab $10 \mathrm{mg} / \mathrm{kg}$ every 3 weeks, nivolumab $1 \mathrm{mg} / \mathrm{kg}$ every 3 weeks plus ipilimumab $3 \mathrm{mg} / \mathrm{kg}$ every 3 weeks, and nivolumab $3 \mathrm{mg} / \mathrm{kg}$ every 3 weeks plus ipilimumab $1 \mathrm{mg} / \mathrm{kg}$ every 3 weeks. The basic information of the five RCTs is summarized in Table 1. The earliest of the five studies was published in 2017. The follow-up has ranged from 7.9 to 28 months, with one study not providing detailed follow-up information (17). Moreover, except for the CheckMate 032 trial that sets up three arms but no placebo/chemotherapy, the others were all two arms and compared immunotherapy with placebo or chemotherapy. There was a high degree of consistency among the patients enrolled in the current analysis: before receiving immunotherapy, the patients were over 18 years and had been diagnosed with unresectable, locally advanced or metastatic GC or including GEJ cancer (GEJC); they also had disease progression or intolerance following at least one first-line chemotherapy. The risk of bias assessment for each study according to seven aspects is shown in Figure 2.

\section{Traditional pairwise comparison meta-analysis of immunotherapy and placebo/chemotherapy}

Due to the limited number of adopted studies, there was only one study of each specific ICI versus placebo/ chemotherapy. Therefore, we classified all ICIs as immunotherapy and compared the immunotherapy with placebo/chemotherapy in the traditional pairwise comparison meta-analysis. On account of there being no comparison with placebo/chemotherapy in the CheckMate 032 trial, traditional meta-analysis adopted four of the five RCTs. For survival outcomes, the result of the meta-analysis indicated no statistical difference in PFS or OS between the immunotherapy and placebo/chemotherapy groups (PFS: $\mathrm{HR}=1.22,95 \%$ CI: $0.75-1.99, \mathrm{P}=0.43$; OS: $\mathrm{HR}=0.87,95 \%$ CI: $0.67-1.13, \mathrm{P}=0.30$ ) (Figure $3 A, B$ ). For ORR outcome, the immunotherapy had no obvious advantage in alleviating disease when compared with placebo/chemotherapy (OR $=1.00$, 95\% CI: 0.25-3.97, P=1.00) (Figure 3C). Additionally, placebo/chemotherapy was not superior to immunotherapy in terms of severe TRAEs (OR $=0.70,95 \%$ CI: 0.21-2.31, $\mathrm{P}=0.56$ ) (Figure $3 D$ ). The above results indicate that aside from severe TRAEs, the traditional meta-analysis did not determine a significant advantage of the immunotherapy over placebo/chemotherapy. 


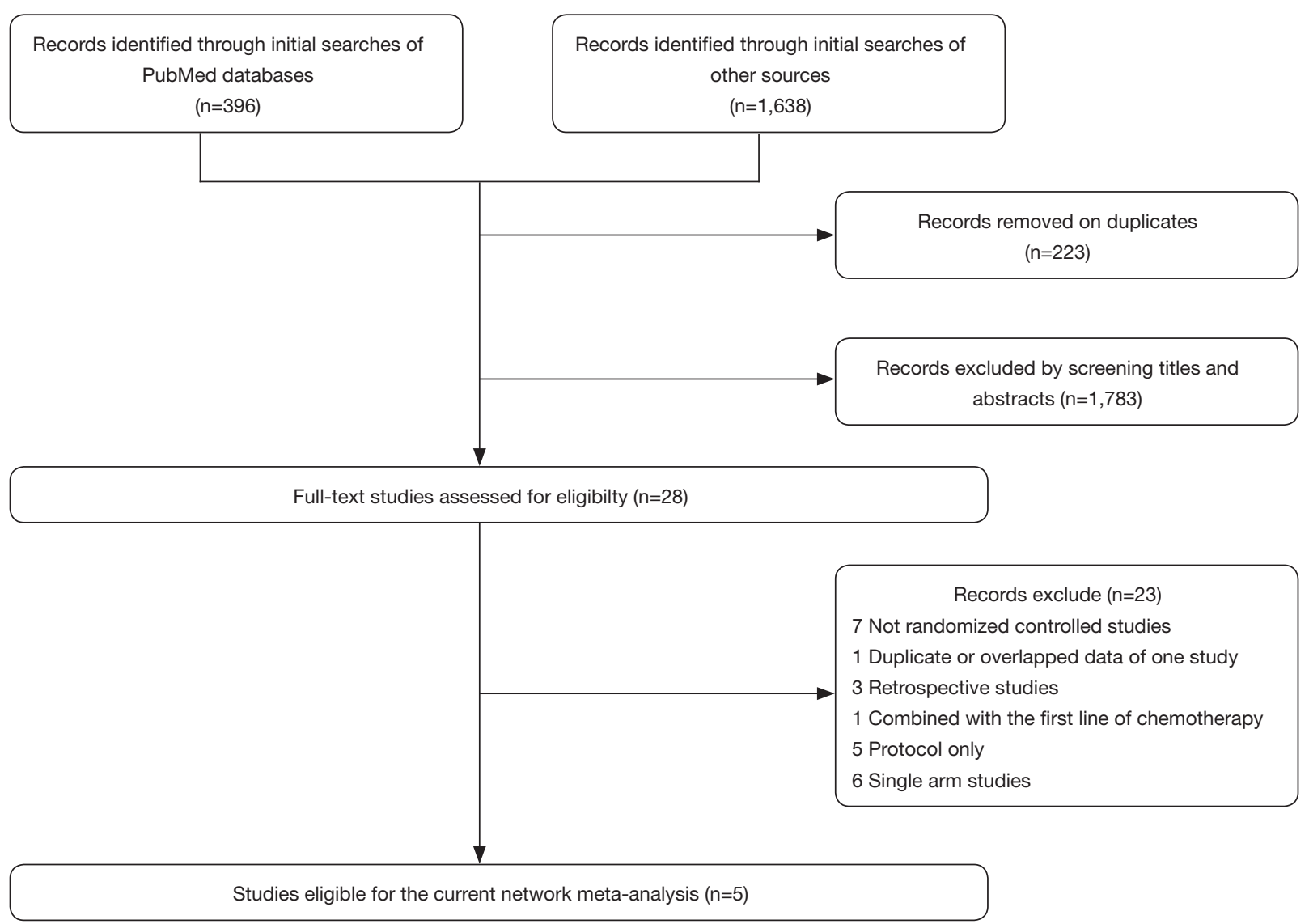

Figure 1 Flow diagram of the study selection of the current meta-analysis.

\section{Network meta-analysis of specific ICIs and placebo/ chemotherapy}

In the current network meta-analysis, we analyzed six different immunotherapeutic treatments and placebo/ chemotherapy (Figure 4A) and different combination of immune targets (Figure $4 B$ ). Due to a large deviation, we did not present the ORR comparisons among different regimens.

For PFS outcome in Figure S1, we observed that nivolumab $1 \mathrm{mg} / \mathrm{kg}$ every 3 weeks plus ipilimumab $3 \mathrm{mg} / \mathrm{kg}$ every 3 weeks (HR $=0.60,95 \%$ CI: $0.49-0.74$ ) and nivolumab $3 \mathrm{mg} / \mathrm{kg}$ every 2 weeks (HR $=0.49,95 \%$ CI: 0.27-0.91) could significantly prolong patients' PFS when compared to placebo/chemotherapy. On the contrary, avelumab $10 \mathrm{mg} / \mathrm{kg}$ every 2 weeks $(\mathrm{HR}=1.57$, 95\% CI: 1.29-1.91), pembrolizumab $200 \mathrm{mg}$ every 3 weeks (HR $=1.49,95 \%$ CI: $1.25-1.77$ ), and ipilimumab $10 \mathrm{mg} / \mathrm{kg}$ every 3 weeks (HR $=1.62,95 \% \mathrm{CI}: 1.03-2.55$ ) led to poorer PFS than placebo/chemotherapy. Moreover, as shown in Figure 5A, we ranked all treatments. We found that nivolumab $1 \mathrm{mg} / \mathrm{kg}$ every 3 weeks plus ipilimumab $3 \mathrm{mg} / \mathrm{kg}$ every 3 weeks $(88.369 \%)$ had the highest probability of prolonging PFS, followed by nivolumab $3 \mathrm{mg} / \mathrm{kg}$ every 3 weeks plus ipilimumab $1 \mathrm{mg} / \mathrm{kg}$ every 3 weeks (81.514\%), and nivolumab $3 \mathrm{mg} / \mathrm{kg}$ every 2 weeks $(77.275 \%)$. In terms of OS outcome, only nivolumab $3 \mathrm{mg} / \mathrm{kg}$ every 2 weeks (HR $=0.62,95 \%$ CI: $0.51-0.76$ ) was observed to be significantly superior to placebo/ chemotherapy and also better than avelumab $10 \mathrm{mg} / \mathrm{kg}$ every 2 weeks, pembrolizumab $200 \mathrm{mg}$ every 3 weeks, and ipilimumab $10 \mathrm{mg} / \mathrm{kg}$ every 3 weeks (Figure S2). Furthermore, nivolumab $3 \mathrm{mg} / \mathrm{kg}$ every 2 weeks $(84.563 \%)$ had the highest probability of prolonging OS, followed by nivolumab $1 \mathrm{mg} / \mathrm{kg}$ every 3 weeks plus ipilimumab $3 \mathrm{mg} / \mathrm{kg}$ every 3 weeks $(84.556 \%)$ and nivolumab $3 \mathrm{mg} / \mathrm{kg}$ every 3 weeks plus ipilimumab $1 \mathrm{mg} / \mathrm{kg}$ every 3 weeks $(54.519 \%)$ (Figure 5B). When focusing on severe TRAEs, we analyzed and ranked it from the perspective of not causing severe TRAEs. As shown in Figure S3, it is 


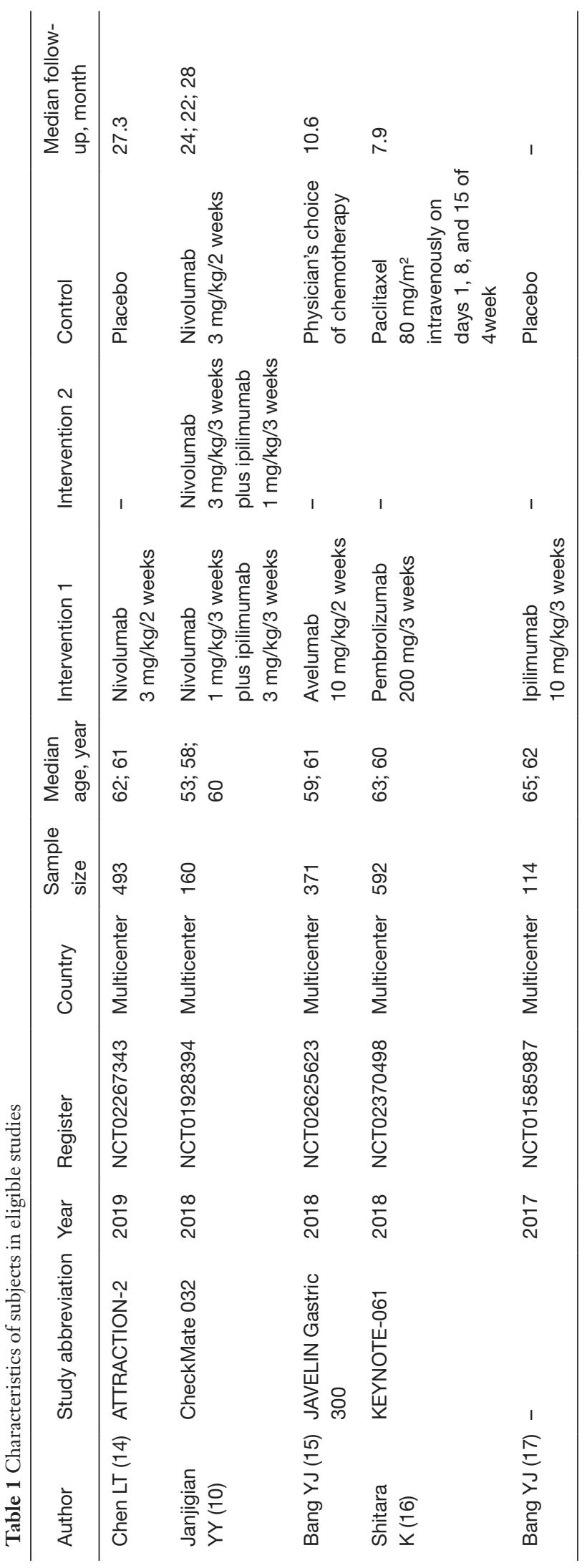

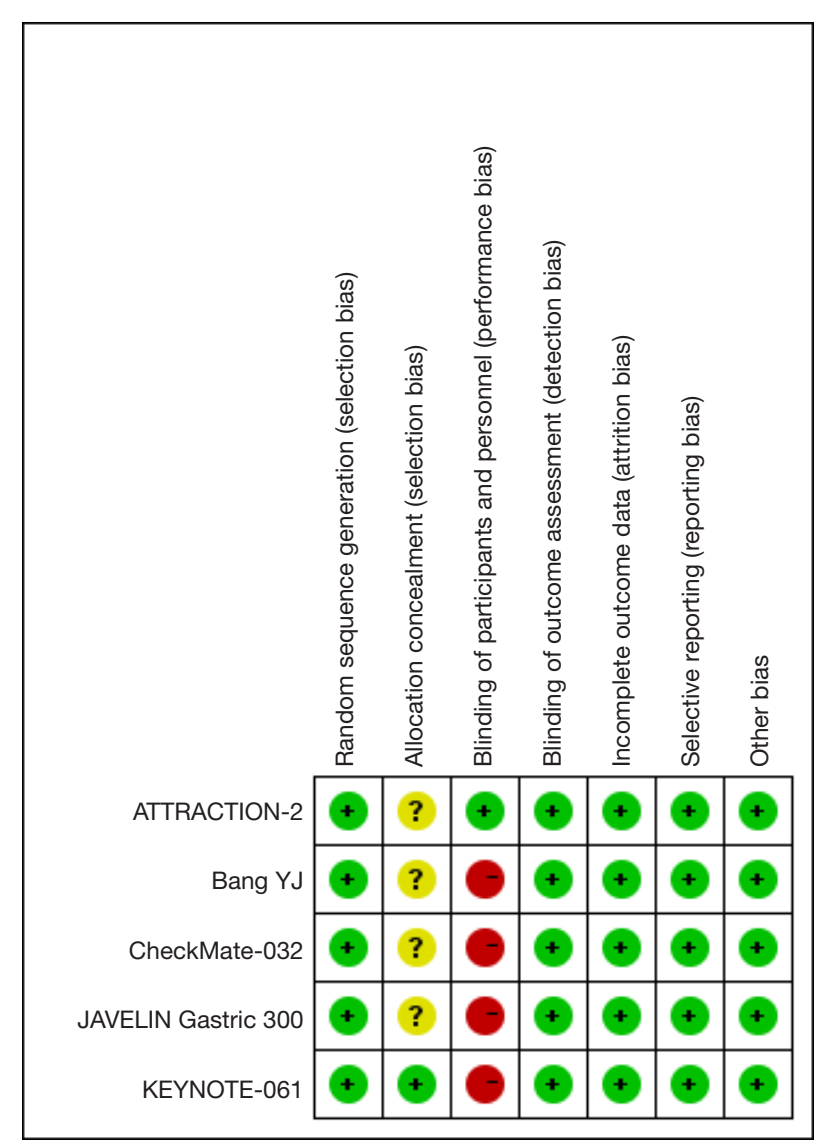

Figure 2 Risk of bias assessment from seven aspects for the adopted studies.

worth noting that placebo/chemotherapy did not show a superior ability to prevent severe TRAEs. Among direct and indirect analyses, avelumab $10 \mathrm{mg} / \mathrm{kg}$ every 2 weeks was the only comparison having a significantly higher OR than ipilimumab $10 \mathrm{mg} / \mathrm{kg}$ every 3 weeks ( $\mathrm{HR}=36.79$, 95\% CI: $1.26-1,066.00)$. It is encouraging that there was no significant difference in the other comparisons. In the rank part, nivolumab $1 \mathrm{mg} / \mathrm{kg}$ every 3 weeks plus ipilimumab $3 \mathrm{mg} / \mathrm{kg}$ every 3 weeks had the lowest probability in preventing severe TRAEs (15.333\%), which might be attributed to the combination of ICIs (Figure 5C).

\section{Network meta-analysis of treatments for different immune targets and placebo/chemotherapy}

After comparing different ICIs, we intended to compare the therapeutic effects of the immune targets of the drugs (Figure 4B). 


\section{A Progression-free survival}

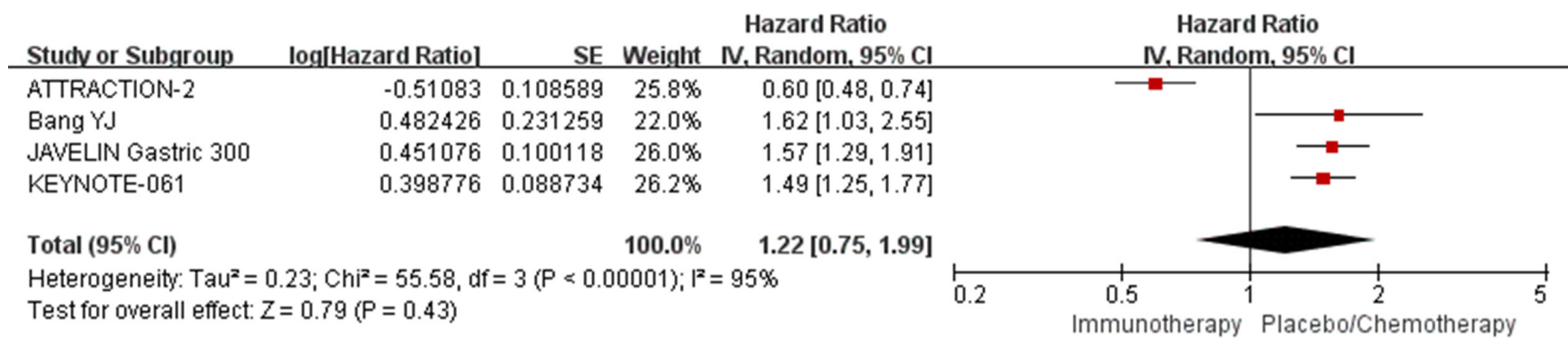

\section{B Overall survival}

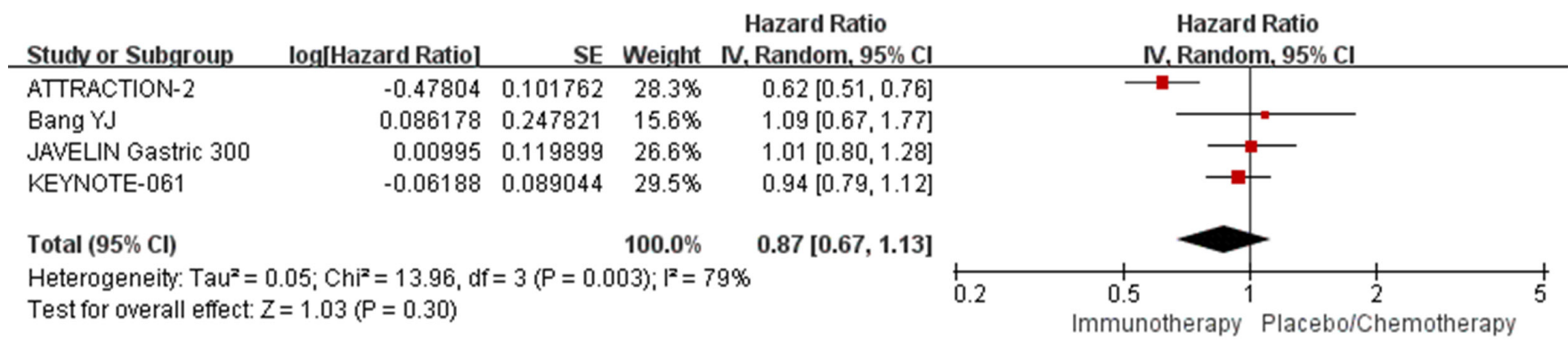

\section{Overall response rate}

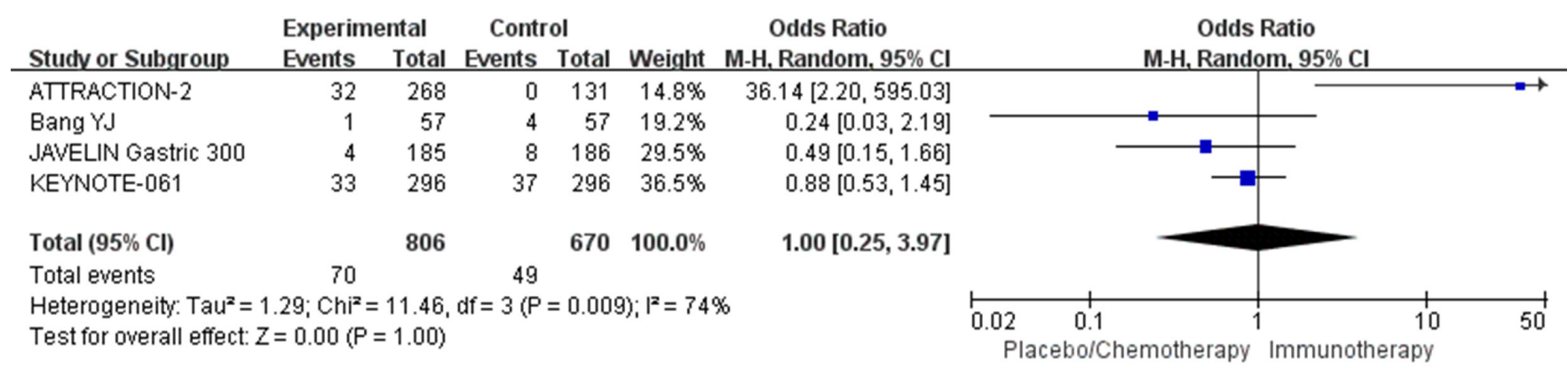

\section{D severe treat-related adverse event}

\begin{tabular}{|c|c|c|c|c|c|c|c|c|c|c|c|}
\hline \multirow[b]{2}{*}{ Study or Subgroup } & \multicolumn{2}{|c|}{ Experimental } & \multicolumn{2}{|c|}{ Control } & \multirow{2}{*}{\multicolumn{2}{|c|}{\begin{tabular}{cc}
\multicolumn{2}{c}{ Odds Ratio } \\
Weight & M-H. Random, $95 \% \mathrm{Cl}$ \\
\end{tabular}}} & \multirow{2}{*}{\multicolumn{5}{|c|}{$\begin{array}{c}\text { Odds Ratio } \\
\text { M-H, Random, } 95 \% \mathrm{Cl}\end{array}$}} \\
\hline & Events & Total & Events & Total & & & & & & & \\
\hline Bang YJ & 13 & 57 & 4 & 57 & $21.5 \%$ & $3.91[1.19,12.87]$ & & & & & \\
\hline KEYNOTE-061 & 42 & 294 & 96 & 276 & $26.4 \%$ & $0.31[0.21,0.47]$ & & & & & \\
\hline Total $(95 \% \mathrm{Cl})$ & & 865 & & 641 & $100.0 \%$ & $0.70[0.21,2.31]$ & & & & & \\
\hline
\end{tabular}

Figure 3 Traditional pairwise comparison meta-analysis between immunotherapy and placebo/chemotherapy: (A) PFS; (B) OS; (C) ORR; (D) severe TRAEs. PFS, progression-free survival; OS, overall survival; ORR, objective response rate; TRAE, treatment-related adverse event. 


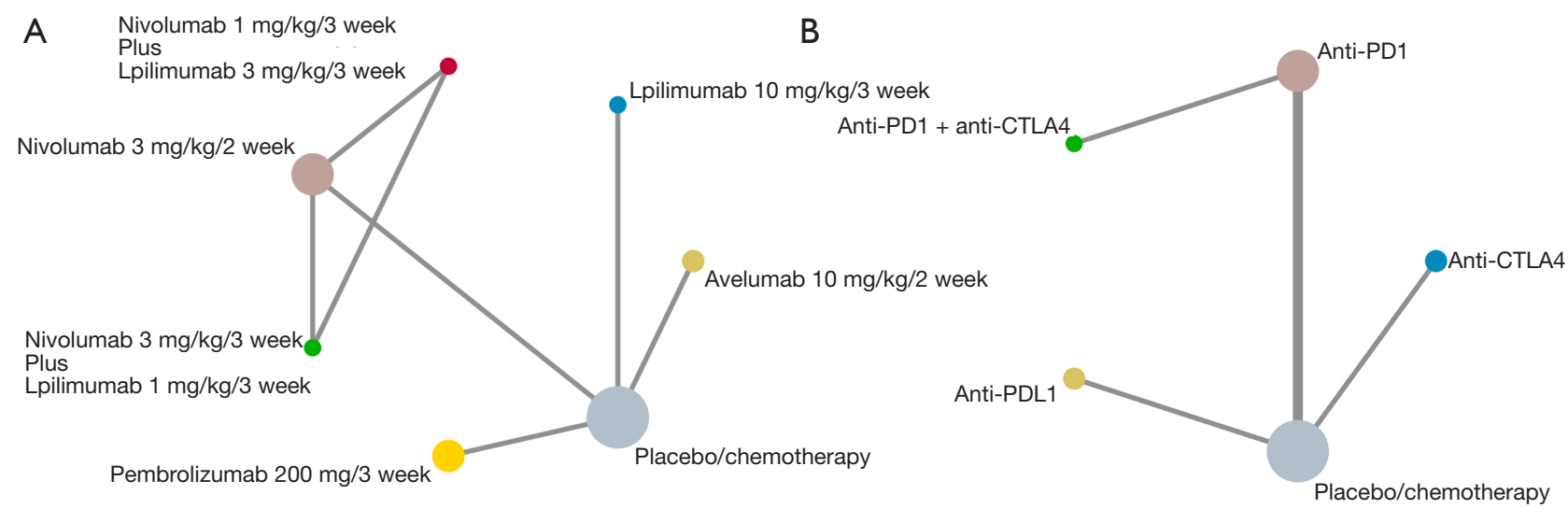

Figure 4 Network plot of comparisons for all interventions adopted in the network meta-analyses: (A) comparison among ICIs and placebo/ chemotherapy; (B) comparisons among ICIs' immune targets and placebo/chemotherapy. The size of each node represents the number of eligible patients and the line thickness shows the number of studies for each comparison. ICI, immune checkpoint inhibitor.

Our results showed that no immune target had a significantly superior ability to prolong GC patients' PFS than placebo/chemotherapy (Figure S4). The latter $(81.952 \%)$ also had the highest probability of prolonging PFS (Figure 5D). Moreover, among the four different immune targets, the anti-PD-1 drug $(69.962 \%)$ and antiPD-1 plus anti-CTLA-4 drug (69.954\%) were more advantageous for prolonging PFS. Differing from PFS, the anti-PD-1 drug (HR =0.78, 95\% CI: 0.69-0.89) was found to have significant advantages in prolonging the OS of GC patients when compared with placebo/chemotherapy (Figure S5). The anti-PD-1 drug (87.472\%) and anti-PD-1 plus anti-CTLA-4 drug (71.955\%) had higher probabilities than placebo/chemotherapy to prolong patients' OS and also were better than the other two immune targets (Figure 5E). When comparing severe TRAEs, no significant difference was found among the four immune targets and placebo/chemotherapy (Figure S6). After ranking, we found that the anti-PD-1 drug $(64.500 \%)$ prevented severe TRAEs better than placebo/chemotherapy, while the anti-PD-1 plus anti-CTLA-4 drug (32.000\%) was worse (Figure $5 F$ ).

\section{Discussion}

This is the first network meta-analysis to study the efficacy and safety of ICIs (PD-1, PD-L1, and CTLA-4 antibodies) as second-line or later treatment for AGC. This article has included a total of five relevant RCTs having 1,730 cases until April 11, 2020. Intervention regimens were categorized according to the clinical trials as follows: nivolumab $1 \mathrm{mg} / \mathrm{kg}$ every 3 weeks plus ipilimumab $3 \mathrm{mg} / \mathrm{kg}$ every 3 weeks, nivolumab $3 \mathrm{mg} / \mathrm{kg}$ every 3 weeks plus ipilimumab $1 \mathrm{mg} / \mathrm{kg}$ every 3 weeks, nivolumab $3 \mathrm{mg} / \mathrm{kg}$ every 2 weeks, pembrolizumab $200 \mathrm{mg}$ every 3 weeks, avelumab $10 \mathrm{mg} / \mathrm{kg}$ every 2 weeks, and ipilimumab $10 \mathrm{mg} / \mathrm{kg}$ every 3 weeks.

In the network analysis, nivolumab $1 \mathrm{mg} / \mathrm{kg}$ every 3 weeks plus ipilimumab $3 \mathrm{mg} / \mathrm{kg}$ every 3 weeks (88.369\%) had the best efficacy and was the most likely to improve PFS but met a high and severe TRAE rate. Nivolumab $3 \mathrm{mg} / \mathrm{kg}$ every 2 weeks was best for OS outcome (84.563\%). The incidence of severe TRAEs of pembrolizumab and avelumab treatment was lower than that of nivolumab and ipilimumab regimens. None of the ICI regimens significantly increased ORR in our traditional pairwise comparison meta-analysis. The ORRs were similar to those in the monotherapy setting, no matter whether the patients had PD-L1+ or PD-L1- tumors. However, in the CheckMate 032 study, combination therapy with different doses of nivolumab plus ipilimumab met higher ORRs in both combination groups than monotherapy (24\% and $8 \%)(18)$. In the KEYNOTE-059 trial, patients with PDL1+ vs. PD-L1- tumors had trends for higher ORRs (19).

The findings of the traditional meta-analysis suggested that anti-PD-1/PD-L1 treatment compared to chemotherapy improved long-term OS clinical benefit and prolonged the duration of response in pretreated advanced or metastatic GC/GEJC patients $(20,21)$. AntiPD-1 therapy may have worked better for PD-L1+ patients; however, it was also effective for PD-L1- patients. The traditional meta-analysis could compare only the advantages 

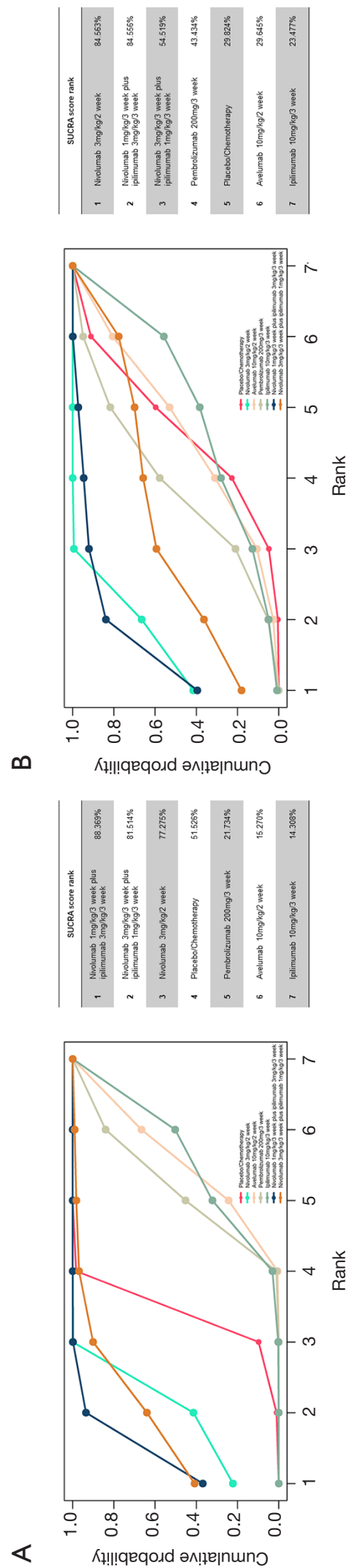
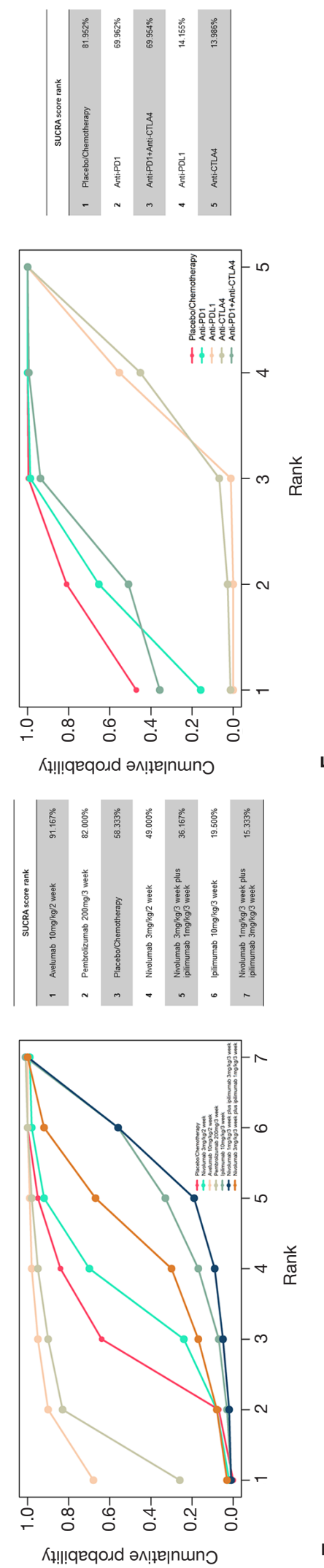
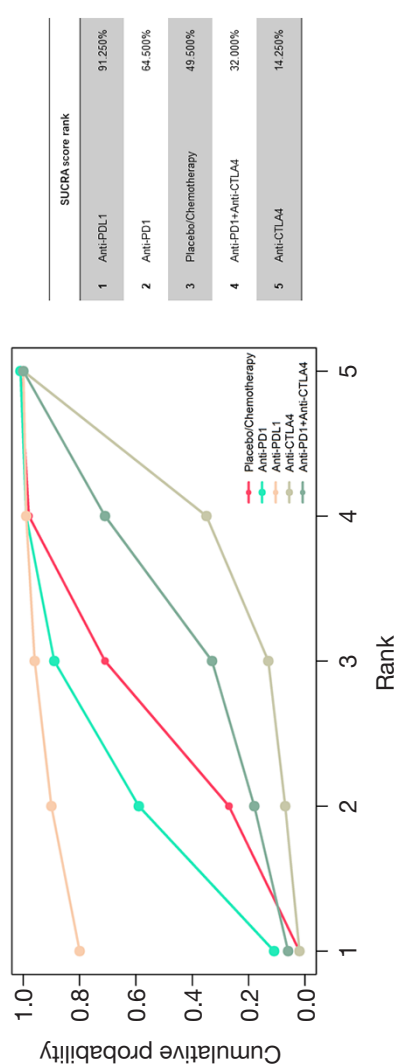

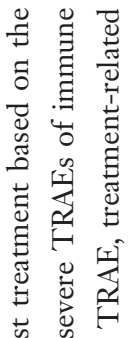

ป

\pm is

का

$\stackrel{5}{\square}$

政寻

ㅁ․ 0

年

음

:

$\pm$

宊

एँ

苛 貝

$\circ$.

ए人.

丩

ᄉł!!!qeqoxd әк!̣e|nunว

อิ

4

药

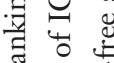

氙爱

矛

㤹

跣岱

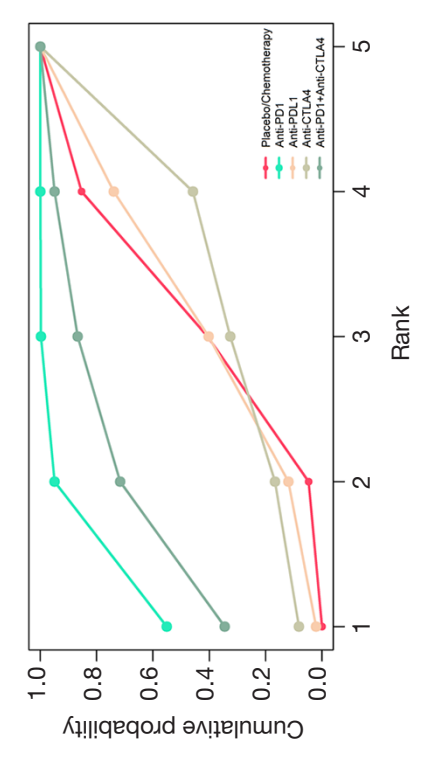

.0

突

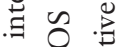

ปี

范光

는

㟧

苋

文

离葑

竝 疍

约 
and disadvantages of immunotherapy with regular chemotherapy or placebo without grouped comparison between the different drugs and regimens (21). Network analysis could determine which specific regimens were relatively effective on PFS, OS, ORR, and TRAEs using direct and indirect comparisons of interventions within RCTs or across multiple treatments. The blinded RCTs included in our study design had high credibility and an improvement over the previous meta-analysis.

The processes that inhibit $\mathrm{T}$ cell function are called immune checkpoint pathways. Better immunotherapies for cancer patients could be achieved if the prevention of immune checkpoint signaling could be identified and the antitumor T-cell functions be reactivated by these pathways (22). GC is considered as a cold type of tumor that usually has a low infiltration of CD8+ T cells, as well as immune-suppressive cells (23). However, some studies suggest that the expression of PD-L1 may improve the prognosis of patients by increasing the proportion of CD8+ TIL. PD-L1 is commonly expressed in GC cells in the range of $17.4-49.1 \%(21,24)$. That is a delightful prospective of ICIs in AGC. Although these therapies are associated with long-lasting response rates, only a subset of patients derives clinical benefit, which varies according to the tumor type. The research for predicting the response to ICIs is a matter of intense investigation as this may contribute toward maximizing disease control, reducing side effects, and minimizing cost. The approval of ICIs for use in GC has pushed immuno-oncology research in this cancer type. In Table 1, we summarize the clinical trials evaluating the safety and efficacy of ICIs in GC.

After pembrolizumab was approved in the United States for PD-L1+ tumors and nivolumab in Japan, third-line (3L) treatment has evolved to include immunotherapy regimens in AGC/GEJC (25). The first study of pembrolizumab, the KEYNOTE-012 study, included 39 patients with recurrent or metastatic PD-L1+ GC/GEJC. The ORR was $22 \%$, the median OS was 11.4 months, and grade $\geq 3$ TRAEs occurred in $13 \%$ of the patients (8). Following these results, pembrolizumab was trialed in patients with GC/GEJC (KEYNOTE-059). This study comprised three cohorts. Cohort 1 represented the largest early-phase trial of ICI in GC/GEJC, enrolling 259 patients who received pembrolizumab monotherapy as $3 \mathrm{~L}$ or later treatment. The ORR was $15.5 \%$ with a trend for higher ORR in PD$\mathrm{L} 1+v s$. PD-L1- tumors (16\% vs. 6\%, respectively). The median OS was 16.3 months $(9,19)$. The ATTRACTION-2 study also revealed that nivolumab administration to
GC patients was associated with improved OS compared to patients treated with placebo regardless of the $\mathrm{PD}$ L1 status $(14,26)$. However, the current literature data regarding the prognosis has shown controversial results (KEYNOTE-062). Pembrolizumab alone or in combination with cisplatin/5-FU vs. cisplatin/5-FU alone in patients with AGC showed that pembrolizumab or chemotherapy brought survival benefit to patients with PD-L1-positive AGC. However, there was no difference in PFS and OS between pembrolizumab plus chemotherapy compared with chemotherapy alone (27). The above illustrates the efficacy of ICI treatment with a certain degree of hysteresis, but it can provide some patients with long-term clinical benefits. Our analysis has shown that anti-PD-1 with or without antiCTLA-4 treatment, compared with placebo/chemotherapy, improved the OS rate but had no advantage in terms of PFS. The number of high-quality studies is limited. In addition, many of them were single-arm studies, and the number of patients was low. This may amplify inaccuracies. High-quality RCT research is needed for verification.

In addition to assessing monotherapy, combination therapy with different doses of nivolumab plus ipilimumab (N1I3: nivolumab $1 \mathrm{mg} / \mathrm{kg}$ plus ipilimumab $3 \mathrm{mg} / \mathrm{kg}$; N3I1: nivolumab $3 \mathrm{mg} / \mathrm{kg}$ plus ipilimumab $1 \mathrm{mg} / \mathrm{kg}$ ) was assessed in patients with AGC in the CheckMate 032 study $(10,18)$. The median OS in the N1I3 and N3I1 subgroups were 6.9 and 4.8 months, respectively. Grade $\geq 3$ TRAEs occurred in $47 \%$ and $27 \%$. In cohort 2 of KEYNOTE-059, 25 patients with HER2-AGC received treatment with $1 \mathrm{~L}$ pembrolizumab in combination with $5-\mathrm{FU} /$ cisplatin chemotherapy. The ORR was $60 \%$, and there was a potential association between PD-L1+ tumors and higher ORR $(69 \%$ and $38 \%$ in patients with PD-L1+ vs. PD-L1tumors, respectively). The median OS was 13.8 months. The incidence of grade $3 / 4$ TRAEs was $76 \%$, which was notably higher than that seen with $1 \mathrm{~L}$ pembrolizumab monotherapy $(23 \%)$. Our network meta-analysis proved that while various combinations of ICI treatment achieved promising results in treating AGC, TRAEs occurred more often with combination therapy than with monotherapy in the multiple treatments. This should be given more attention.

Key problems in immunosuppressive therapy need to be solved urgently. At present, the question remains of how to correctly screen the subset of patients that would respond well to ICIs. The indicators of immunosuppressive treatment in the population reported in the literature include mainly PD-1/PD-L1 expression level, microsatellite 
instability (MSI) level, and tumor mutation load burden. However, no precise screening standard or guideline has been released. The expression of PD-L1 was high in MSI-H GC; MSI-H may induce innate antitumor immune responses and make tumors more sensitive to immune checkpoint blockades $(28,29)$. Results from the KEYNOTE-061 study showed that pembrolizumab was significantly safer than the standard second-line paclitaxel treatment, and subgroup analysis showed that patients with high PD-L1 expression or MSI-H benefitted from the pembrolizumab treatment (16). Some meta-analyses found that the efficacy of anti-PD-1 therapy in MSI-H patients was significantly better than that of microsatellite stable, which could increase ORR to 3.40 times and disease control rate to 2.26 times (21). In addition, the efficacy of pembrolizumab was also related to circulating tumor DNA (ctDNA). Compared to patients with a low ctDNA mutation load, patients with a high mutation load had higher ORRs (30).

More than half of the patients receiving ICIs developed TRAEs, but the probability of developing grade $\geq 3$ TRAEs was low and the incidence of treatment leading to death was almost zero $(15,31,32)$. Therefore, ICI treatment can be considered as being safe. How to predict and prevent TRAEs from tumor immunotherapy is challenging. The side effects of tumor immunotherapy include mainly fatigue, skin ulcers, immune dermatitis, immune colitis, immune hepatitis, immune thyroiditis, and immune nephritis. Tumor pseudo- and hyper-progression was also recently reported to be one of the important TRAEs of immunotherapy (33). How to simultaneously block proteins involved in immune system regulation (such as TNF, IL-6) can separate the efficacy and toxicity of combined immunotherapy but needs further study.

The merit of the network meta-analysis of this study was that we compared the efficacy and safety of seven therapeutic regimens, including different combinations of four ICIs referring to three immune targets for AGC. In contrast, many clinical trials have directly compared only one or two ICIs with traditional chemotherapy or placebo. Moreover, the trials selected in this network meta-analysis were all RCTs with big samples and had high evidencebased value. Using direct and indirect comparisons of interventions within RCTs or across multiple treatments, it is also the first network meta-analysis to determine which specific regimens are relatively effective on PFS, OS, ORR, and TRAEs. There are also some limitations in this network meta-analysis that should be noted. Firstly, only five RCTs studies were included, which limited the sample size. Due to the limited literature, many indicators might have some heterogeneity. Meanwhile, there is insufficient data on ORR, which leads to results that have low reliability. Further tests should be determined to optimize the result. The lack of standardized agents in chemotherapy or placebos may also affect the reliability and validity of our results. Furthermore, our study only compared the therapeutic value of partial PD-1, PD-L1, and CTLA-4 inhibitors. Atezolizumab and durvalumab were not enrolled in the comparison as we failed to retrieve related RCTs.

Herein, we focused on the therapeutic effects of ICIs on AGC patients and carried out network meta-analysis to compare each ICI and immune target. We found that nivolumab with or without ipilimumab had a significantly better ability than the placebos or traditional chemotherapy to prolong patients' PFS and OS without causing significant severe TRAEs. Furthermore, in immune target analyses, anti-PD-1 with or without anti-CTLA-4 drugs were confirmed as having OS benefit but no PFS benefit. Therefore, according to the regimens adopted in the five RCTs, we recommend nivolumab $3 \mathrm{mg} / \mathrm{kg}$ every 2 weeks or nivolumab $1 \mathrm{mg} / \mathrm{kg}$ every 3 weeks plus ipilimumab $3 \mathrm{mg} / \mathrm{kg}$ every 3 weeks as the preferred regimen.

\section{Acknowledgments}

The authors appreciate the help and discussions of all members of the Department of Oncology Surgery of First Hospital of China Medical University.

Funding: This study was supported by grants from the National Natural Science Foundation of China (Grant No. 81772549).

\section{Footnote}

Reporting Checklist: The authors have completed the PRISMA-NMA reporting checklist. Available at http:// dx.doi.org/10.21037/atm-20-6639

Conflicts of Interest: All authors have completed the ICMJE uniform disclosure form (available at http://dx.doi. org/10.21037/atm-20-6639). The authors have no conflicts of interest to declare.

Ethical Statement: The authors are accountable for all aspects of the work in ensuring that questions related to the accuracy or integrity of any part of the work are 
appropriately investigated and resolved.

Open Access Statement: This is an Open Access article distributed in accordance with the Creative Commons Attribution-NonCommercial-NoDerivs 4.0 International License (CC BY-NC-ND 4.0), which permits the noncommercial replication and distribution of the article with the strict proviso that no changes or edits are made and the original work is properly cited (including links to both the formal publication through the relevant DOI and the license). See: https://creativecommons.org/licenses/by-nc-nd/4.0/.

\section{References}

1. Bray F, Ferlay J, Soerjomataram I, et al. Global cancer statistics 2018: GLOBOCAN estimates of incidence and mortality worldwide for 36 cancers in 185 countries. CA Cancer J Clin 2018;68:394-424.

2. Feng RM, Zong YN, Cao SM, et al. Current cancer situation in China: good or bad news from the 2018 Global Cancer Statistics? Cancer Commun (Lond) 2019;39:22.

3. Allemani C, Matsuda T, Di Carlo V, et al. Global surveillance of trends in cancer survival 2000-14 (CONCORD-3): analysis of individual records for 37 513025 patients diagnosed with one of 18 cancers from 322 population-based registries in 71 countries. Lancet 2018;391:1023-75.

4. Wagner AD, Syn NL, Moehler M, et al. Chemotherapy for advanced gastric cancer. Cochrane Database Syst Rev 2017;8:CD004064.

5. Lordick F, Janjigian YY. Clinical impact of tumour biology in the management of gastroesophageal cancer. Nat Rev Clin Oncol 2016;13:348-60.

6. Taieb J, Moehler M, Boku N, et al. Evolution of checkpoint inhibitors for the treatment of metastatic gastric cancers: current status and future perspectives. Cancer Treat Rev 2018;66:104-13.

7. Kubota Y, Kawazoe A, Sasaki A, et al. The impact of molecular subtype on efficacy of chemotherapy and checkpoint inhibition in advanced gastric cancer. Clin Cancer Res 2020;26:3784-90.

8. Muro K, Chung HC, Shankaran V, et al. Pembrolizumab for patients with PD-L1-positive advanced gastric cancer (KEYNOTE-012): a multicentre, open-label, phase 1b trial. Lancet Oncol 2016;17:717-26.

9. Fuchs CS, Doi T, Jang RW, et al. Safety and efficacy of pembrolizumab monotherapy in patients with previously treated advanced gastric and gastroesophageal junction cancer: phase 2 clinical KEYNOTE-059 trial. JAMA Oncol 2018;4:e180013.

10. Janjigian YY, Bendell J, Calvo E, et al. CheckMate-032 study: efficacy and safety of nivolumab and nivolumab plus ipilimumab in patients with metastatic esophagogastric cancer. J Clin Oncol 2018;36:2836-44.

11. Rouse B, Chaimani A, Li T. Network meta-analysis: an introduction for clinicians. Intern Emerg Med 2017;12:103-11.

12. Higgins JP, Altman DG, Gotzsche PC, et al. The Cochrane Collaboration's tool for assessing risk of bias in randomised trials. BMJ 2011;343:d5928.

13. Zhao TT, Xu H, Xu HM, et al. The efficacy and safety of targeted therapy with or without chemotherapy in advanced gastric cancer treatment: a network meta-analysis of well-designed randomized controlled trials. Gastric Cancer 2018;21:361-71.

14. Chen LT, Satoh T, Ryu MH, et al. A phase 3 study of nivolumab in previously treated advanced gastric or gastroesophageal junction cancer (ATTRACTION-2): 2-year update data. Gastric Cancer 2020;23:510-9.

15. Bang YJ, Ruiz EY, Van Cutsem E, et al. Phase III, randomised trial of avelumab versus physician's choice of chemotherapy as third-line treatment of patients with advanced gastric or gastro-oesophageal junction cancer: primary analysis of JAVELIN Gastric 300. Ann Oncol 2018;29:2052-60.

16. Shitara K, Ozguroglu M, Bang YJ, et al. Pembrolizumab versus paclitaxel for previously treated, advanced gastric or gastro-oesophageal junction cancer (KEYNOTE-061): a randomised, open-label, controlled, phase 3 trial. Lancet 2018;392:123-33.

17. Bang YJ, Cho JY, Kim YH, et al. Efficacy of sequential ipilimumab monotherapy versus best supportive care for unresectable locally advanced/metastatic gastric or gastroesophageal junction cancer. Clin Cancer Res 2017;23:5671-8.

18. Sun S, Kim JJ, Ko YH. CheckMate-032 Study: promising efficacy with nivolumab-based immunotherapy in pretreated esophagogastric cancer. J Thorac Dis 2019;11:S394-5.

19. Bang YJ, Kang YK, Catenacci DV, et al. Pembrolizumab alone or in combination with chemotherapy as firstline therapy for patients with advanced gastric or gastroesophageal junction adenocarcinoma: results from the phase II nonrandomized KEYNOTE-059 study. Gastric Cancer 2019;22:828-37.

20. Ninomiya K, Oze I, Kato Y, et al. Influence of age on the 
efficacy of immune checkpoint inhibitors in advanced cancers: a systematic review and meta-analysis. Acta Oncol 2020;59:249-56.

21. Chen C, Zhang F, Zhou N, et al. Efficacy and safety of immune checkpoint inhibitors in advanced gastric or gastroesophageal junction cancer: a systematic review and meta-analysis. Oncoimmunology 2019;8:e1581547.

22. Lin R, Zhang H, Yuan Y, et al. Fatty acid oxidation controls CD8(+) tissue-resident memory T-cell survival in gastric adenocarcinoma. Cancer Immunol Res 2020;8:479-92.

23. Pernot $S$, Terme M, Radosevic-Robin N, et al. Infiltrating and peripheral immune cell analysis in advanced gastric cancer according to the Lauren classification and its prognostic significance. Gastric Cancer 2020;23:73-81.

24. Ralph C, Elkord E, Burt DJ, et al. Modulation of lymphocyte regulation for cancer therapy: a phase II trial of tremelimumab in advanced gastric and esophageal adenocarcinoma. Clin Cancer Res 2010;16:1662-72.

25. Eto S, Yoshikawa K, Nishi M, et al. Programmed cell death protein 1 expression is an independent prognostic factor in gastric cancer after curative resection. Gastric Cancer 2016;19:466-71.

26. Satoh T, Kang YK, Chao Y, et al. Exploratory subgroup analysis of patients with prior trastuzumab use in the ATTRACTION-2 trial: a randomized phase III clinical trial investigating the efficacy and safety of nivolumab in patients with advanced gastric/gastroesophageal junction cancer. Gastric Cancer 2020;23:143-53.

27. Tabernero J, Cutsem EV, Bang Y-J, et al. Pembrolizumab with or without chemotherapy versus chemotherapy for

Cite this article as: Pan S, Li K, Huang B, Huang J, Xu H, Zhu Z. Efficacy and safety of immune checkpoint inhibitors in gastric cancer: a network meta-analysis of well-designed randomized controlled trials. Ann Transl Med 2021;9(4):290. doi: 10.21037/atm-20-6639 advanced gastric or gastroesophageal junction (G/GEJ) adenocarcinoma: the phase III KEYNOTE-062 study. J Clin Oncol 2019;37:LBA4007.

28. Choi YY, Kim H, Shin SJ, et al. Microsatellite instability and programmed cell death-ligand 1 expression in stage II/III gastric cancer: post hoc analysis of the CLASSIC randomized controlled study. Ann Surg 2019;270:309-16.

29. Danaher P, Warren S, Ong S, et al. A gene expression assay for simultaneous measurement of microsatellite instability and anti-tumor immune activity. J Immunother Cancer 2019;7:15.

30. Ishiba T, Hoffmann AC, Usher J, et al. Frequencies and expression levels of programmed death ligand 1 (PD-L1) in circulating tumor RNA (ctRNA) in various cancer types. Biochem Biophys Res Commun 2018;500:621-5.

31. Kawazoe A, Yamaguchi K, Yasui H, et al. Safety and efficacy of pembrolizumab in combination with S-1 plus oxaliplatin as a first-line treatment in patients with advanced gastric/gastroesophageal junction cancer: Cohort 1 data from the KEYNOTE-659 phase IIb study. Eur J Cancer 2020;129:97-106.

32. Huang J, Mo H, Zhang W, et al. Promising efficacy of SHR-1210, a novel anti-programmed cell death 1 antibody, in patients with advanced gastric and gastroesophageal junction cancer in China. Cancer 2019;125:742-9.

33. Togasaki K, Sukawa Y, Kanai T, et al. Clinical efficacy of immune checkpoint inhibitors in the treatment of unresectable advanced or recurrent gastric cancer: an evidence-based review of therapies. Onco Targets Ther 2018;11:8239-50. 